\section{Vigilancia del consumo de antimicrobianos en hospitales de México: situación actual y guía práctica para su implementación}

\author{
Odalis Rodríguez-Ganen ${ }^{1}$ y \\ Juan Asbun-Bojalil ${ }^{1}$
}

Forma de citar: Rodríguez-Ganen O, Asbun-Bojalil J. Vigilancia del consumo de antimicrobianos en hospitales de México: situación actual y guía práctica para su implementación. Rev Panam Salud Publica. 2012;32(5):381-6.

\section{SINOPSIS}

En México se han constatado un elevado consumo de antibióticos, su uso irracional en la atención primaria y altas tasas de resistencia en bacterias causantes de infecciones nosocomiales. Se hace necesario revisar la metodología recomendada para informar el consumo de antimicrobianos, de manera que la cuantificación se realice mediante estudios de utilización de medicamentos. Estos estudios, que permiten conocer los medicamentos utilizados en el hospital, requieren de una fuente de datos de consumo de antimicrobianos accesible y con el menor riesgo de sesgos, un sistema de identificación único para los fármacos y la adopción de unidades de medida extrapolables. Se propone usar la metodología elaborada por el Centro Colaborador de la Organización Mundial de la Salud para la Metodología Estadística de Medicamentos, basada en la clasificación anatómica, terapéutica y química (ATC) y la dosis diaria definida (DDD). A pesar de sus limitaciones, el empleo de esta metodología para el monitoreo local del consumo de antimicrobianos permite detectar diferencias en los patrones de uso respecto a otras instituciones $y$, sobre todo, las tendencias dentro de una misma institución. Esto ayudaría a alertar oportunamente sobre posibles problemas en la utilización de antimicrobianos y la necesidad de implementar intervenciones específicas.

Palabras clave: antibacterianos; farmacorresistencia bacteriana; utilización de medicamentos; hospitales; México.

\footnotetext{
Escuela Superior de Medicina, Sección Estudios de Posgrado e Investigación, Instituto Politécnico Nacional, México, D.F., México. La correspondencia se debe enviar a Juan Asbun-Bojalil. Correo electrónico: jasbunb@ipn.mx, juanasbun@yahoo.com.mx
}

El uso inadecuado de medicamentos antimicrobianos se ha relacionado con problemas en la atención de salud (1), en particular, con la resistencia bacteriana a los antibióticos. Esta resistencia lleva no solo al aumento de la morbilidad y la mortalidad, sino también a estancias hospitalarias más prolongadas, lo que incrementa los costos de hospitalización y de la atención sanitaria (2-4).

Según la Organización Mundial de la Salud (OMS), las estadísticas sobre el consumo de los medicamentos antimicrobianos en los hospitales, además de reflejar la presión selectiva sobre los microorganismos, permiten evaluar comparativamente las instituciones de uno o varios países y establecer directrices para la prescripción y la vigilancia del uso de estos fármacos (1). Por ello, se ha implementado la vigilancia sistemática de los antimicrobianos en redes hospitalarias de los Estados Unidos de América (5) y varios países de Europa $(6,7)$.

En México, se han constatado tanto el elevado consumo de antibióticos (8) como su uso irracional en la atención primaria $(9,10)$ y las altas tasas de resistencia en bacterias causantes de infecciones nosocomiales $(11,12)$. Estos hallazgos generan dudas sobre la correcta prescripción y el uso adecuado de antimicrobianos en los hospitales del país y llevaron a revisar las investigaciones sobre la práctica de prescripción y el consumo de antimicrobianos en ellos.

Por ello, se consideró necesario revisar la metodología recomendada para informar el consumo de antimicrobianos, de manera que la cuantificación se realice mediante estudios de utilización de medicamentos (13), basados en la clasificación anatómica, terapéutica y química (ATC) y la dosis diaria definida (DDD) (14).

Según el sistema ATC, propuesto por el Centro Colaborador de la OMS para la Metodología Estadística de Medicamentos (CCDEM) para la clasificación universal de los fármacos, estos se identifican mediante letras y números de acuerdo a los cinco niveles de codificación: 1) el anatómico, compuesto por 14 grupos principales según el órgano o sistema sobre el que actúa el fármaco; 2) el subgrupo terapéutico principal; 3) el subgrupo terapéutico farmacológico; 4) el subgrupo químico terapéutico farmacológico; y 5) el código del producto según la Denominación Común Internacional, recomendada por la OMS para la identificación internacional de los medicamentos $(13,15)$.

La DDD, por su parte, es la unidad de medición del consumo de medicamentos más ampliamente utilizada por su universalidad $(6,13,14,16,17)$, aunque se han empleado otras, como la dosis diaria prescrita (DDP) y los días de tratamiento (DDT), entre otras (5, $18,19)$. Independientemente de la unidad de medida escogida, el uso de las formas de presentación habitua- 
les -número de envases o unidades de dosificación, como tabletas, viales, gotas y concentración, entre otras- introduce una gran variabilidad en la cuantificación del consumo de medicamentos. Por ello, es preferible utilizar como unidad de medida la DDD según la definición del CCDEM, es decir, la dosis media diaria recomendada de un fármaco cuando se utiliza para su indicación principal, expresada como el peso de la sustancia activa $(13,16,17)$.

Para conocer los antecedentes respecto a las metodologías empleadas para informar el consumo de antimicrobianos, se revisaron los artículos publicados en los principales índices médicos: Pubmed, Medline, Embase, SciELO, Redalyc, LILACS, Biblioteca Virtual de Salud y Latindex. Se utilizó el descriptor "México" combinado con los términos "utilización de medicamentos", "agentes antibacterianos", "farmacorresistencia microbiana" y "hospital", y esos mismos términos en inglés ("Mexico", "drug utilization", "antibacterial agent", "bacterial resistance" y "hospital").

\section{Prescripción y utilización de antimicrobianos en hospitales de México}

Existen pocos informes relacionados con el uso y el consumo de antimicrobianos en este país. De los informes encontrados, los que estudian el consumo carecen total o parcialmente de una metodología estándar que permita la extrapolación y la comparación de sus resultados.

El tratamiento inadecuado con antibióticos se asocia significativamente con el aumento de la mortalidad, como lo demostró el seguimiento farmacológico de los casos críticos de neumonía asociada a la ventilación mecánica en cuatro unidades de cuidados intensivos que identificó, además, una fuerte asociación entre la resistencia a los antibióticos y la prescripción inadecuada (20).

Si bien los estudios en niños no han demostrado que la profilaxis antimicrobiana disminuya significativamente el riesgo de infección quirúrgica, su uso inadecuado eleva innecesariamente los costos y aumenta la presión de selección de los microorganismos nosocomiales. La revisión de las prescripciones de los casos con profilaxis quirúrgica en un hospital pediátrico constató que en $39 \%$ de los casos se incumplieron las recomendaciones internacionales para el uso profiláctico de antimicrobianos en cirugía, en particular, se administró el antimicrobiano una vez finalizada la cirugía (21).

El seguimiento de los casos de neumonía adquirida en la comunidad al ingreso en un hospital privado mostró que $47,5 \%$ de los tratamientos no era efectivo contra gérmenes atípicos y en $20 \%$ era cuestionable la efectividad contra neumococo, lo que evidenció la falta de adherencia a las guías de práctica clínica (22).

\section{Vigilancia del consumo de medicamentos antimicrobianos}

Se pueden y deben tomar medidas para mejorar la calidad de la prescripción de medicamentos anti- microbianos. Un estudio realizado en seis hospitales de tercer nivel de Ciudad de México informó el uso de hasta 216,1 DDD/100 camas-día de cefalosporinas (23); esto contrasta con el consumo de 80 DDD/100 camas-día para todos los antimicrobianos, observado en el servicio de cirugía general de un hospital canadiense (16). Se debe aclarar, no obstante, que en el estudio mexicano no se utilizó la clasificación ATC y la información capturada data de hace más de 15 años.

En otro estudio basado en cinco años de monitoreo del consumo de antimicrobianos en una unidad de cuidados intensivos de un hospital del sector público se demostró que gracias a ese seguimiento y a las intervenciones derivadas de este, las prescripciones empíricas adecuadas mejoraron de $50 \%$ a $70 \%$ (24). Sin embargo, no se utilizó el sistema de clasificación y medición ATC/DDD en el diseño del estudio.

\section{Consideraciones sobre la vigilancia de antimicrobianos en hospitales}

De cinco estudios encontrados sobre utilización de medicamentos, tres analizaron la práctica de prescripción de antimicrobianos y en todos los casos se observó un uso inadecuado de estos medicamentos (20-22). De los dos estudios que analizaron el consumo (23, 24), solo uno (23) siguió parcialmente la metodología de la OMS, lo que imposibilita la comparación de sus resultados con los de otros hospitales.

En la bibliografía encontrada, se documentan diversas intervenciones dirigidas a fortalecer la vigilancia de la prescripción y el uso de los antimicrobianos.

Por ejemplo, en la Norma Oficial Mexicana para la vigilancia epidemiológica, prevención y control de las infecciones nosocomiales (25) se prevé que las unidades de vigilancia epidemiológica hospitalarias apoyen al subcomité de control de antimicrobianos en el seguimiento del uso de estos fármacos y de la resistencia antimicrobiana.

Además, en un trabajo se menciona la existencia de FARMAC (26), un sistema de información y vigilancia de la prescripción de antibióticos que permite consultar la información actualizada y analizar las tendencias y patrones del uso de los agentes antimicrobianos. Por otro lado, está la propuesta de lineamientos de regulación y promoción para el uso adecuado de antibióticos en México (27), que prevé la cuantificación de las prescripciones de antibióticos en los hospitales, como parte de la implementación de sistemas eficientes de vigilancia epidemiológica. Sin embargo, no se encontró información sobre los resultados o el impacto de estas dos iniciativas.

A todo esto debe añadirse que en las políticas farmacéuticas (28) y de salud (29) de México apenas se discute el problema del uso racional de antibióticos.

Todo lo anterior indica que a pesar de las medidas tomadas, no se vislumbran mejoras inmediatas para lograr una vigilancia efectiva del uso de antimicrobianos en el país. Uno de los elementos clave para lograr estos objetivos es contar con una metodología 
estándar para los estudios de consumo, que permita establecer comparaciones entre los hospitales y estimule la adopción de respuestas locales.

\section{VIGILANCIA DEL CONSUMO HOSPITALARIO DE ANTIMICROBIANOS MEDIANTE ESTUDIOS DE UTILIZACIÓN DE MEDICAMENTOS}

Aunque no existe aún una medición específica para cuantificar el uso de antibióticos (5), el CCDEM promueve la realización de estudios de utilización de medicamentos $(13,19)$, que permiten conocer los medicamentos utilizados en el hospital y las cantidades compradas, dispensadas, prescritas o consumidas. De esta forma, se pueden detectar malas prácticas e identificar los factores responsables, y con esta información diseñar intervenciones efectivas y evaluar sus resultados. Para desarrollar este tipo de estudios, se requieren tres elementos: una fuente de datos de consumo de antimicrobianos accesible y con el menor riesgo de sesgos, un sistema de identificación único para los fármacos y la adopción de unidades de medida extrapolables.

\section{Fuentes de datos de consumo de antimicrobianos en el hospital}

Como fuentes de datos de consumo se pueden utilizar: a) las prescripciones (indicaciones médicas o recetas) registradas en el expediente clínico, b) los antibióticos consumidos por el paciente según lo reflejado en los informes de enfermería, c) la dispensación intrahospitalaria según los registros de la farmacia, d) los informes de los comités de control de medicamentos, y e) los registros de compra.

Se debe tener en cuenta, no obstante, que los registros de compra no reflejan la administración real del fármaco al paciente y que, aunque los datos de prescripción se aproximan más al consumo real, sus resultados pueden no ser extrapolables o el proceso de obtención puede prolongarse tanto que se pierde la razón del estudio. Las dispensaciones de la farmacia, sobre todo las basadas en sistemas de distribución por dosis unitaria, establecen más fidedignamente la exposición del paciente al fármaco.

Para la identificación de los fármacos se optó por la clasificación ATC. Como unidad de medida del consumo de antimicrobianos se escogió la DDD, ya sea como indicador en términos absolutos o como tasa en relación con indicadores hospitalarios, por ejemplo, DDD/100 camas-día (13, 15-17)

\section{Ejemplo de la cuantificación del consumo de antibióticos en un hospital}

Se partirá de un caso hipotético en el que se requiere identificar los $\beta$-lactámicos inyectables de mayor consumo y se dispone de las dispensaciones hechas por la farmacia del hospital en el quinquenio 2006-2010.

Para responder a este objetivo, se seguiría el siguiente procedimiento:

a) La información de las dispensaciones intrahospitalarias se asientan en un modelo de captación de datos (por ejemplo, $\mathrm{Excel}^{\odot}$ ) que debe incluir: el código único de identificación (asignado por la industria o el hospital), el nombre comercial, la denominación común internacional (de no existir, se debe agregar), la presentación farmacéutica (tabletas, ampolletas, etc.), el contenido de principio activo ( $\mathrm{mg}$, $\mathrm{g}$, unidades) y la cantidad dispensada.

b) Se realiza la clasificación de los antimicrobianos — según las guías para la clasificación ATC - y la asignación de la DDD (30), para lo cual se puede usar el motor especializado de búsqueda en línea del sitio del CCDEM (15). Como las guías indican que el primer nivel de clasificación ATC para los antimicrobianos tiene el código $J$ (antiinfectivos de uso sistémico), se asignará ese código y a partir de ahí se asignará el segundo nivel de clasificación según los subgrupos terapéuticos como 01 (antibacterianos de uso sistémico). Así se logrará obtener el resto del código de clasificación ATC hasta llegar al quinto nivel (ver ejemplo para ceftriaxona en el cuadro 1).

c) Una vez completada la clasificación ATC, el motor de búsqueda muestra la DDD recomendada por la OMS. En el caso de la ceftriaxona, la clasificación ATC sería J01DD04 y la DDD recomendada para la vía parenteral sería de $2 \mathrm{~g}$.

d) El consumo hospitalario, expresado por lo general en unidades dispensadas, se deben convertir a gramos a partir del número de unidades vendidas de un mismo fármaco, según la clasificación ATC, y el contenido de sustancia activa.

e) Con la DDD recomendada por la OMS y las estadísticas hospitalarias (número de camas y porcentaje

CUADRO 1. Ejemplo de la clasificación anatómica, terapéutica y química (ATC) para ceftriaxona, estructurada en los cinco niveles

\begin{tabular}{|c|c|c|c|}
\hline $\begin{array}{l}\text { Nivel de } \\
\text { clasificación }\end{array}$ & Categoría ATC & Descripción & Clasificación del nivel \\
\hline 1 & J & $\begin{array}{l}\text { Antiinfecciosos generales de uso } \\
\text { sistémico }\end{array}$ & Grupo anatómico principal \\
\hline 2 & J01 & Antibacterianos de uso sistémico & Subgrupo terapéutico \\
\hline 3 & J01D & $\begin{array}{l}\text { Otros antibacterianos } \beta \text {-lactámicos, } \\
\text { cefalosporinas }\end{array}$ & Subgrupo terapéutico farmacológico \\
\hline 4 & J01DD & Cefalosporinas de tercera generación & Subgrupo químico, terapéutico, farmacológico \\
\hline 5 & J01DD04 & Ceftriaxona & Subgrupo por principio activo \\
\hline
\end{tabular}


de ocupación) se puede obtener el consumo en DDD/100 camas-día. ${ }^{2}$ El cálculo se realiza según la siguiente fórmula $(13,16,17)$ :

$$
\mathrm{DDD} / 100 \text { camas-día }=\frac{a}{b} \times \frac{100}{t \times n \times O c}
$$

donde,

a: consumo del antibacteriano durante el período analizado, en gramos

$b$ : DDD recomendada del fármaco por la OMS en el período analizado, gramos

$t$ : tiempo analizado, en días

$n$ : número de camas del hospital

Oc: porcentaje de ocupación del hospital

De esta forma, el consumo de ceftriaxona (DDD recomendada por la OMS: $2 \mathrm{~g}$ ) durante un año en un hospital de 144 camas y una ocupación media de 76,8\%, en el que se dispensaron $9694 \mathrm{~g}$, sería de 12,01 DDD/100 camas-día. Esto significa que por cada 100 camas del hospital, 12 pacientes consumieron $2 \mathrm{~g}$ de ceftriaxona diariamente.

f) Al analizar las DDD obtenidas por niveles de clasificación (figura 1) se pudo comprobar que las cefalosporinas fueron los fármacos más consumidos en el hospital estudiado, muy por encima de la penicilina, y que hacia 2010 se elevó ligeramente el uso de carbapenemos. Además del alto consumo de ceftriaxona y cefalotina, se observó que al final del período aumentó el consumo de cefepima (figura 2). Esto indica que las próximas intervenciones en este hospital se deben encausar hacia el control del uso de ceftriaxona, cefepima y carbapenemos.

El cálculo general del consumo - por grupos e individualmente- de los antimicrobianos de mayor utilización, importancia estratégica o sometidos a intervención puede ayudar a identificar las causas de las desviaciones en su prescripción (32). Estos datos pueden correlacionarse con las estadísticas del movimiento hospitalario (por ejemplo, los egresos de las unidades de vigilancia epidemiológica hospitalarias, y la morbilidad y la mortalidad por enfermedades infecciosas, entre otras) o de la adherencia a las guías de práctica clínica.

Aunque el impacto del consumo sobre la susceptibilidad bacteriana ha sido objeto de estudio $(18,33-$ $35)$, la ecología de la resistencia a antimicrobianos es un fenómeno complejo, por lo que para su análisis se requiere de una metodología sólida y la integración de especialistas en microbiología, epidemiología, infectología, control de expedientes e, incluso, estadística hospitalaria $(18,32,34)$. Una alternativa más sencilla al alcance del profesional farmacéutico es clasificar los antimicrobianos según sus indicaciones clínicas y luego correlacionar su consumo con la susceptibilidad de los gérmenes identificados en el hospital (33-35).

2 El cálculo puede realizarse manualmente o mediante la herramienta ABC Calc (versión 3.1), disponible en el sitio de Internet de la Sociedad Europea de Microbiología y Enfermedades Infecciosas (31).
FIGURA 1. Tendencias del consumo intrahospitalario de antibacterianos $\beta$-lactámicos (penicilinas y otros $\beta$-lactámicos seleccionados), según el tercer nivel de la clasificación anatómica, terapéutica y química (ATC), durante el quinquenio 2006-2010

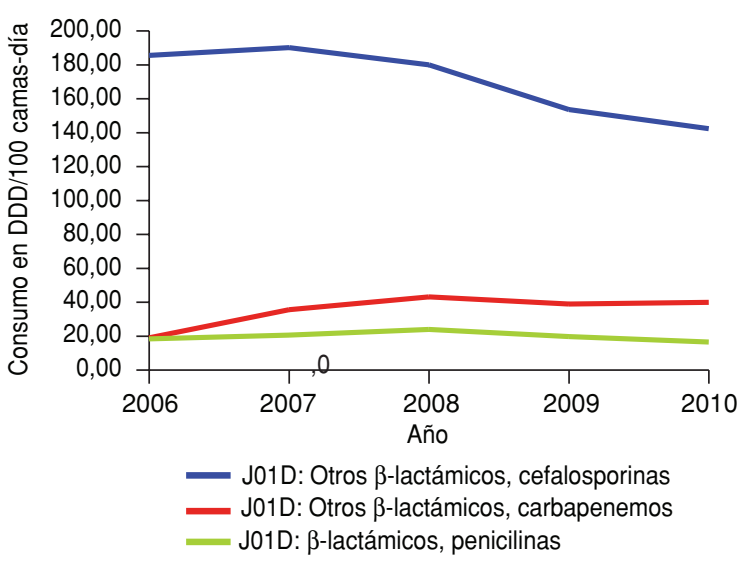

DDD: dosis diaria definida.

FIGURA 2. Tendencias del consumo intrahospitalario de cefalosporinas, según el quinto nivel de clasificación anatómica, terapéutica y química (ATC), durante el quinquenio 2006-2010

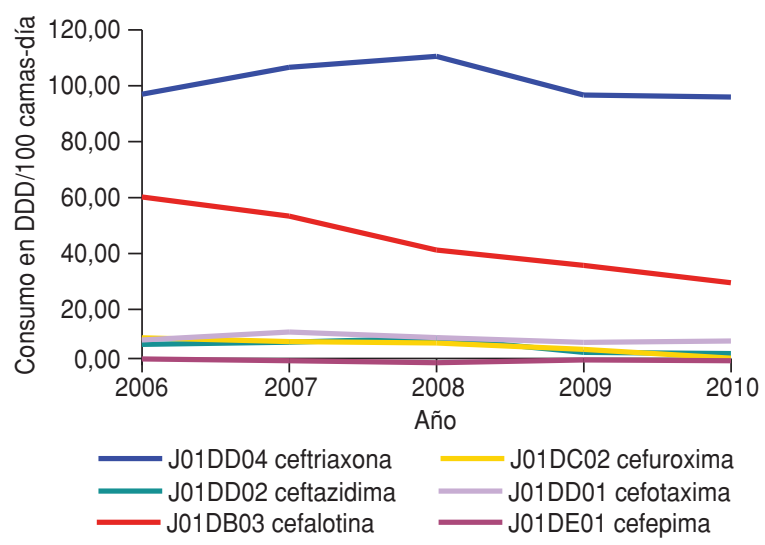

DDD: dosis diaria definida.

\section{CONSIDERACIONES FINALES}

Según la OMS, la presentación —-tanto a los médicos que prescriben como a las autoridades sanitarias y políticas - de la información sobre la vigilancia del consumo de antimicrobianos puede constituir el primer paso para crear conciencia de la importancia del cuidado en la prescripción de estos fármacos. Combinada con el seguimiento de la resistencia a antimicrobianos, esta medida puede ayudar a establecer políticas de utilización de antibióticos y guías locales de tratamiento empírico, además de permitir explicar los casos presentados, realizar predicciones o generar hipótesis de trabajo.

Si bien lo ideal es mantener el seguimiento multicéntrico y sistemático de la resistencia detectada a antimicrobianos $(1,5-7)$, la aplicación de estudios de 
utilización local con la metodología basada en la clasificación ATC y la DDD puede generar las primeras señales de alerta sobre el uso irracional de estos medicamentos, lo que permitiría controlar oportunamente el problema $(17,32-35)$.

Todas las unidades de medición del consumo de antimicrobianos tienen ventajas y desventajas. Aunque la DDP puede representar fielmente el consumo de antimicrobianos y tiene en cuenta las características individuales del paciente — como el peso, la edad o las consideraciones farmacocinéticas-, a diferencia de la DDD puede variar en el tiempo y en dependencia del lugar, lo que hace difícil las comparaciones. Por su parte, los DDT no consideran las dosis empleadas (5) y su cálculo requiere de mayor tiempo que la DDD y la $\operatorname{DDP}(5,17,19)$.

También la DDD tiene limitaciones como unidad de medida de consumo de medicamentos. Entre ellas destacan las siguientes:

a) El valor de la DDD no necesariamente se corresponde con la DDP o los DDT. Algunos autores encontraron que la medición del consumo de ampicilina en DDD (sola o asociada con sulvactam) sobreestimó hasta en tres veces los DDT y la DDP $(5,19)$.

b) No se han establecido valores de DDD específicas para niños y se ha demostrado que al utilizar la DDD en casos pediátricos se sobreestima el consumo al utilizar dosis elevadas y se subestima al usar dosis bajas (36).

c) Una misma DDD puede expresar muy diferentes grados de exposición a los antibióticos en distintas áreas de hospitalización, instituciones o áreas geográficas, en dependencia del tipo de dosificación empleada y su duración $(17,19,37)$.

A estas limitaciones, independientemente de la unidad de medida utilizada, pueden sumarse las dificultades en la extracción de los datos de consumo desde el sistema de inventarios de la farmacia hospitalaria, así como su conversión según la clasificación ATC y las DDD, lo que podría afectar a la precisión de estos estudios. Esto, no obstante, se podría solucionar si se aplicara al cuadro básico nacional de medicamentos la clasificación ATC, como un código único de identificación nacional, y la cuantificación en DDD, según lo recomendado por la OMS. Además, habría que adecuar el inventario local de las farmacias hospitalarias mediante sistemas de informatización basados en la clasificación ATC y las DDD.

A pesar de las limitaciones mencionadas, el monitoreo local del consumo de antimicrobianos mediante la metodología basada en la clasificación ATC y las DDD permite detectar diferencias en los patrones de uso respecto a otras instituciones $\mathrm{y}$, sobre todo, las tendencias dentro de una misma institución. Esto ayudaría a alertar oportunamente sobre posibles problemas en la utilización de estos fármacos y la necesidad de implementar intervenciones específicas.

Para complementar la vigilancia, se sugiere instaurar la receta intrahospitalaria como mecanismo de control y generación de información, establecer el seguimiento farmacoterapéutico de las prescripciones por parte de un profesional farmacéutico y crear indicadores que reflejen la calidad de la prescripción.

Agradecimientos. A la memoria de Aline Espinosa-Fernández. Se agradece el apoyo brindado a esta investigación por el Consejo Nacional de Ciencia y Tecnología (CONACyT) y la Comisión de Operación y Fomento de Actividades Académicas del Instituto Politécnico Nacional (COFFAA-IPN), a través del Programa Integral de Fortalecimiento Institucional (PIFI). Los autores agradecen a Pedro López-Sánchez los útiles comentarios hechos a este trabajo.

\section{SYNOPSIS}

\section{Surveillance of antimicrobial drug use in Mexican hospitals: current situation and practical guidelines for its implementation}

Antibiotic use in Mexico is characterized by high levels of consumption, irrational use in primary care services, and high rates of bacterial resistance that cause hospital infections. Changes are needed in the recommended method for reporting antimicrobial drug use, so that quantification can be carried out through drug utilization studies. Such studies, which make it possible to know which drugs are used in hospitals, require an accessible source of data on antimicrobial drug use with the least risk of biases, a single drug identification system, and the adoption of units of measure that can be extrapolated. The methodology developed by the WHO Collaborating Centre for Drug Statistics Methodology, based on the anatomical, therapeutic, and chemical classification (ATC) system and the daily defined dosage (DDD), is proposed. Despite its limitations, employing this methodology to monitor local antimicrobial drug use will make it possible to detect differences in usage patterns among institutions, and, especially, trends within a given institution. This would facilitate timely alerts about potential problems related to the use of antimicrobial drugs and the need for specific interventions.

Keywords: antibacterial agents; drug resistance, bacterial; drug utilization; hospitals; Mexico.

\section{REFERENCIAS}

1. Organización Mundial de la Salud. Farmacorresistencia. Vigilancia del uso de los antimicrobianos. Ginebra: OMS 2010. Disponible en: http:/ / www.who. int/drugresistance/surveillance use/es / Acceso el 10 de abril de 2010.

2. Stone PW, Braccia D, Larson E. Systematic review of economic analyses of health care-associate infections. Amer J Infect Control. 2005;33(9):501-9.

3. Rosenthal VD, Maki DG, Mehta A, Álvarez-Moreno C, Leblebicioglu $\mathrm{H}$, 
Higuera $F$, et al. International Nosocomial Infection Control Consortium report, data summary for 2002-2007, issued January 2008. Amer J Infect Control. 2008;36(9):627-37.

4. Rosenthal V, Maki D, Salomao R, Álvarez-Moreno C, Mehta Y, et al. Device-associated nosocomial infections in 55 intensive care units of 8 developing countries. Ann Intern Med. 2006;145:582-91.

5. Polk R, Fox C, Mahoney A, Letcavage J, MacDougall C. Measurement of adult antibacterial drug use in 130 US hospitals: comparison of defined daily dose and days of therapy. Clin Infect Dis. 2007:44:664-70.

6. Health Protection Surveillance Centre. Consumption of antibiotics in public acute hospitals in Ireland. Data to end of second cuarter of 2012. Disponible en: http://www.hpsc.ie/hpsc/A-Z/ MicrobiologyAntimicrobialResistance/ EuropeanSurveillanceofAntimicrobial ConsumptionESAC/SurveillanceRe ports/File,13678,en.pdf Acceso el 12 de noviembre de 2012

7. Mac Kenzie F, Monnet D, Gould I, on behalf of the ARPAC Steering Group. Relationship between the number of different antibiotics use. J Antimicrob Chemother. 2006;58:657-60.

8. Wirtz VJ, Dreser A, Gonzales R. Trends in antibiotic utilization in eight Latin American countries, 1997-2007. Rev Panam Salud Publica. 2010;27(3):219-25.

9. Dreser A, Wirtz VJ, Corbett KK, Echániz G. Uso de antibióticos en México: revisión de problemas y políticas. Salud Publica Mex. 2008;50(4):S480-7.

10. Wirtz VJ, Reich MR, Leyva R, Dreser A. A systematic review of research on access and use in Mexico 1990-2004. Salud Publica Mex. 2008;50(4):470-9.

11. Velázquez $\mathrm{N}$, Vigueras JC, Escalona G, Arellano J, Giono S, Nava M. Resistencia a linezolid en Staphylococcus aureus resistente a meticilina y enterococos con elevada resistencia a aminoglucósidos en un hospital pediátrico de tercer nivel. Bol Med Hosp Infant Mex. 2010;67:19-26.

12. Casanova LJ, Castañón JA, León MA, Becerra JJ, Calyeca MV, Franco AE, et al. Respiratory tract fluid microbiology in an intensive care unit. Rev Med Inst Mex Seguro Soc. 2008;46(3):329-38.

13. Luna A. Farmacoepidemiología. Estudios de utilización de medicamentos. Parte I: Concepto y metodología. Seguim Farmacoter. 2004;2(3):129-36.

14. Organización Mundial de la Salud. Comités de farmacoterapia. Guía práctica. Ginebra: OMS; 2004.

15. WHO Colaborating Centre for Drug Statistics Methodology. ATC/DDD Index 2010. Oslo: Norwegian Institute of Publ Health; 2009. Disponible en: http:// www.whocc.no/news/403.html Acceso el 25 de septiembre de 2012.

16. Hutchinson J, Patrick D, Marra F, Helen $\mathrm{N}$, Bowie R, Heule L, et al. Measure- ment of antibiotic consumption: a practical guide to the use of the Anatomical Therapeutic Chemical classification and Defined Daily Dose system methodology in Canada. Can J Infect Dis. 2004;15(1):29-35.

17. Natch S. Collecting, converting, and making sense of hospital antimicrobial consumption data. En: Gould IM, van der Meer JWM, eds. Antibiotic policies. Fighting resistance. New York: Kluwer Academic/Plenum Publishers; 2005. Pp. 67-74.

18. Cantó R, Cobo J. Consumo de antimicrobianos y resistencia en el hospital: una relación difícil de medir y compleja de interpretar. Enferm Infecc Microbiol Clin. 2009;27(8):437-40.

19. Muller A, Monnet D, Talon D, Hénon $\mathrm{T}$, Bertrand X. Discrepancies between prescribed daily doses and WHO defined daily doses of antibacterials at a university hospital. Br J Clin Pharmacol. 2006;61(5):585-91.

20. Zaidi M, Sifuentes J, Rolón AL, Vázquez G, Rosado R, Sánchez M, et al. Inadequate therapy and antibiotic resistance. Risk factors for mortality in the intensive care unit. Arch Med Res. 2002;33(3):290-4.

21. Méndez MA, Díaz RD, Ramírez JA Miranda MG. Uso de antimicrobianos profilácticos en cirugía en un hospital pediátrico de tercer nivel. Enferm Infecc Microbiol. 2005;25(1): Disponible en: http://www.amimc.org.mx/ revista/2005/25-1/uso_antimicro biano.htm Acceso el 12 de noviembre de 2012.

22. Chávez E, Tovar A, Pérez JR. Evaluación de la acción terapéutica por distintos médicos de neumonía adquirida en la comunidad en un hospital privado de la Ciudad de México. Med Int Mex. 2010;26(1):11-6.

23. Benavides L, Aldama AL, Vázquez HJ. Vigilancia de los niveles de uso de antibióticos y perfiles de resistencia bacteriana en hospitales de tercer nivel de la Ciudad de México. Salud Publica Mex. 2011;47(3):219-26.

24. Rodríguez-Badillo $\mathrm{R}$, Castorena AG, González DF, Suárez VM, Arroyo ES, Moncada BD, et al. Programa de monitoreo bacteriológico y de regulación de uso de antibióticos. Experiencia en una unidad de cuidados intensivos. Rev Asoc Mex Med Crit Ter Intensiva. 2011;25(2):87-96.

25. México, Secretaría de Salud. Norma Oficial Mexicana NOM-045-SSA2-2005, para la vigilancia epidemiológica, prevención y control de las infecciones nosocomiales. México, D.F.: Secretaría de Salud; 2009.

26. Medina F, Navarrete S, Ávila C, Santos JI. FARMAC: a program designed for monitoring the prescription of antimicrobials in hospitals. Gac Med Mex. 2000;136(2):107-11.

27. Instituto Nacional de Salud Pública. Medicamentos en salud pública: uso, acceso y resistencia antimicrobiana. Regulación y promoción para el uso adecuado de antibióticos en México. Propuesta de lineamientos para la acción. México, D.F.: Instituto Nacional de Salud Pública; 2009.

28. Comisión Federal para la Protección Contra Riesgos Sanitarios. Hacia una política farmacéutica integral para México. México, D.F.: Secretaría de Salud; 2005.

29. México, Secretaría de Salud. Programa Nacional de Salud 2007-2012. México, D.F.: Secretaría de Salud; 2007.

30. WHO Collaborating Centre for Drug Statistics Methodology. Guidelines for ATC classification and DDD assignment 2010. 13th edition. Oslo: Norwegian Institute of Publ Health; 2009.

31. European Society of Microbiology and Infection Diseases. ABC Calc. Vers 3.1. Copenhague: ESCMID; 2012. Disponible en: http://www.escmid.org/ research_projects/study_groups/es gap/abc_calc/ Acceso el 25 de septiembre de 2012

32. Rodríguez J, Paño R, Álvarez L, Asencio A, Calvo E, Cercenado E, et al. Programas de optimización de uso de antimicrobianos (PROA) en hospitales españoles: documento de consenso GEIH-SEIMC, SEFH y SEMPSPH. Enferm Infecc Microbiol Clin. 2012;30(1):22. e1-23.

33. Maortua H, Canut A, Ibáñez B, Martínez D, Domingo MJ, Labora A. Relación entre la resistencia bacteriana intrahospitalaria y el consumo de antimicrobianos durante un período de 13 años. Enferm Infecc Microbiol Clin. 2009;27(8): 441-8.

34. Goryachkina K, Babak S, Burbello A Wettemark B, Bergman U. Quality use of medicines: a new method of combining antibiotic consumption and sensitivity data-application in a Russian hospital. Pharmacoepidemiol Drug Saf. 2008;17:636-44.

35. Velickovic R, Petrovic J, Kocic B, Antic S, Mitic R. Analysis of antibiotic utilization and bacterial resistance changes in a surgical clinic of Clinical Centre, Nis. J Clin Pharm Ther. 2012;37:32-6.

36. Liem $T$, Heerdink E, Egberts A Rademaker C. Quantifying antibiotic use in paediatrics: a proposal for neonatal DDDs. Eur J Clin Microbiol Infect Dis. 2010;29:1301-3.

37. Krivoy N, El-Ahal WA, Bar-Lavie Y, Haddad S. Antibiotic prescription and cost patterns in a general intensive care unit. Pharmacy Practice. 2007;5(2):67-73.

Manuscrito recibido el 6 de diciembre de 2011. Aceptado para publicación, tras revisión, el 30 de julio de 2012. 